\title{
Prognóstico da esquizofrenia
}

\section{Paulo R M enezes}

Departamento de Medicina Preventiva, Faculdade de Medicina da Universidade de São Paulo

A investigação do prognóstico da esquizofrenia é fundamental para a prática clínica, para o planejamento de serviços de saúde e para a pesquisa científica. A literatura internacional apresenta um grande número de trabalhos sobre o prognóstico da esquizofrenia, e é necessário considerar diversos aspectos metodológicos para que seja feita uma interpretação adequada dos resultados de cada estudo específico. Esses aspectos incluem o processo de seleção dos sujeitos, as definições de prognóstico adotadas e os procedimentos de avaliação e seguimento.

A seleção da amostra determinará em que extensão os achados de um estudo particular podem ser generalizados, e devem ser levados em consideração os critérios diagnósticos adotados para a inclusão dos sujeitos (critérios mais restritivos, como o DSMIV, tendem a selecionar pacientes com prognóstico menos favorável do que critérios mais inclusivos), a forma de recrutamento dos mesmos (amostras de regiões geográficas incluem um espectro maior de pacientes, em relação ao prognóstico, que amostras de serviços específicos) e o grau de cronicidade da amostra investigada (pacientes com longo tempo de doença são aqueles que já apresentaram prognóstico ruim).

Estudos sobre o curso e prognóstico da esquizofrenia também diferem quanto ao tipo de seguimento: retrospectivo, prospectivo ou uma combinação de ambos. Estudos retrospectivos usam informações já existentes - que nem sempre são de boa qualidade - ou que podem não estar disponíveis. Por outro lado, podem ser o único meio de investigar desfechos em longo prazo da esquizofrenia, como em um período de 20 ou 30 anos. Estudos prospectivos fornecem informação de melhor qualidade, mas sua realização é mais complexa, onerosa e demorada.

Existem amplas variações entre os estudos na duração do seguimento, com alguns voltados para o prognóstico em curto prazo, de um a cinco anos, ${ }^{1-5}$ e outros para o prognóstico em longo prazo. ${ }^{6-8}$ Os pacientes esquizofrênicos tendem a mostrar maior variabilidade na morbidade clínica durante os primeiros cinco anos de doença e depois disso há uma tendência à estabilização em seu estado, seja favorável ou desfavorável.

Dois estudos sobre o prognóstico da esquizofrenia, organizados pela Organização Mundial de Saúde (OMS), merecem destaque por suas características metodológicas: o "International Pilot Study of Schizophrenia" (IPSS) e o "Determinants of Outcome of Severe Mental Disorders" (Dosmed). ${ }^{2}$ Ambos apresentaram desenho de coorte prospectivo, utilizaram amostras com grande número de pacientes, provenientes de regiões geográficas definidas em diversos centros em diferentes países, e fizeram as ava- liações por meio de instrumentos padronizados para a inclusão e para as avaliações de seguimento. Grande parte do conhecimento atual sobre o prognóstico da esquizofrenia provém de publicações originadas desses dois estudos.

\section{Dimensões de prognóstico}

É possível subdividir o prognóstico da esquizofrenia em múltiplas dimensões e perspectivas, como psicopatologia, funcionamento social, uso de serviços psiquiátricos ou mortalidade, cada uma com sua importância e significado próprios. Cada componente está só parcialmente relacionado aos outros e tem diferentes associações com variáveis preditivas.

Uma proporção substancial de pacientes que têm um episódio esquizofrênico apresentará sintomas psiquiátricos contínua ou intermitentemente durante o curso de sua doença. A tendência atual tem sido considerar os sintomas positivos (delírios, alucinações, desagregação do pensamento) e negativos (embotamento afetivo, retraimento social) separadamente, porque eles podem representar diferentes dimensões de prognóstico. A ocorrência de sintomas positivos em pacientes esquizofrênicos após a alta hospitalar é elevada, ${ }^{3,9-10}$ com índices variando entre $15 \%$ e $75 \%$, dependendo principalmente da proporção de pacientes com longo tempo de doença na amostra estudada. Observa-se também variação similar na proporção de pacientes que apresentam sintomas negativos durante o curso da doença., ${ }^{3,9}$

Uma das principais dimensões do prognóstico da esquizofrenia é o prejuízo no ajustamento social, de tal forma que o DSMIV requer a presença dessa característica por boa parte do curso da doença para que seja feito o diagnóstico de esquizofrenia. De maneira global, os estudos mostram que há um curso heterogêneo do funcionamento social na esquizofrenia, com cerca de 1/3 dos pacientes funcionando bem, outro 1/3 em um nível intermediário de ajustamento social e um grupo de tamanho semelhante que desenvolve graves incapacidades no funcionamento social. ${ }^{4,11} \mathrm{Na}$ maior parte dos trabalhos, os pacientes que tiveram sua primeira internação no início do estudo evoluíram melhor que aqueles com internações psiquiátricas prévias. Alguns pacientes apresentam flutuações em seu nível de ajustamento social ao longo do tempo, enquanto outros permanecem em níveis estáveis de funcionamento. ${ }^{1,12}$ De Jong et $\mathrm{al}^{11}$ mostraram que a incapacidade em áreas especificas de funcionamento social parece desenvolver-se ao longo de linhas hierárquicas, primeiro aparecendo nos contatos sociais periféricos, tais como desempenho no trabalho, e então progre- 
dindo em direção a áreas internas do funcionamento, eventualmente afetando o cuidado pessoal.

Outro aspecto de prognóstico freqüentemente encontrado na literatura é o número e duração das hospitalizações após a avaliação inicial. A vantagem de usar esse índice é sua confiabilidade e fácil obtenção. Estima-se que cerca de $80 \%$ dos pacientes com esquizofrenia são internados em enfermaria psiquiátrica ao menos uma vez ao longo da vida, e observa-se uma correlação razoável entre recaída e retorno à hospitalização. Entretanto, nas últimas três décadas houve uma mudança no modelo de cuidado aos pacientes psiquiátricos, com ênfase em uma abordagem baseada na comunidade e importante diminuição no número de internações. Muitos pacientes que agora vivem na comunidade estão gravemente incapacitados, mostram baixos níveis de funcionamento social e têm sintomas psicóticos. A admissão hospitalar está mais claramente influenciada por fatores externos à doença, tais como políticas de internação e disponibilidade de leitos em hospitais psiquiátricos. Assim, uma tendência mais recente tem sido avaliar o risco de readmissão hospitalar usando o tempo de sobrevivência na comunidade entre duas hospitalizações consecutivas. ${ }^{13-15}$

A mortalidade na esquizofrenia é outra dimensão importante do prognóstico que não pode ser negligenciada. Altas taxas de mortalidade entre pacientes esquizofrênicos, comparadas às taxas da população geral, têm sido relatadas, sendo as taxas de suicídio particularmente altas, cerca de 20 vezes maiores que as observadas na população geral. ${ }^{16-18}$ No passado, essas taxas altas foram atribuídas às condições de vida precárias encontradas nos asilos, e atualmente parecem estar associadas com o processo de transferência do cuidado para a comunidade, onde muitos pacientes não têm suporte social adequado, como habitação, renda e cuidado da saúde, e estão mais expostos ao abuso de substâncias. Os dados sobre mortalidade podem ajudar a identificar grupos de alto risco, a monitorar mudanças nos modelos de tratamento e de cuidado ao longo do tempo, a planejar estratégias de intervenção para prevenir mortes prematuras e a avaliar sua eficácia.

\section{Um estudo de prognóstico após a hospitalização no Brasil}

No Brasil foi realizada uma investigação para examinar os desfechos clínico e social, o uso de serviços e a mortalidade dos pacientes com esquizofrenia, dois anos após terem sido internados em hospitais psiquiátricos. Outro objetivo foi estudar quais os fatores preditores desses desfechos. ${ }^{19-21} \mathrm{O}$ estudo foi realizado em uma área definida de captação da cidade de São Paulo e teve um desenho prospectivo, com um período de seguimento de 2 anos a partir da inclusão dos pacientes. Foram incluídos indivíduos com psicoses funcionais não-afetivas consecutivamente internados em hospitais psiquiátricos. Foram usados instrumentos padronizados para a avaliação do estado psicopatológico, dos níveis de ajustamento social e do uso de serviços. A amostra foi composta por 69 homens e 55 mulheres, com média de idade de 32,6 anos e duração média da doença de 8,3 anos: 86 sujeitos $(69,3 \%)$ preencheram critérios do DSM-III-R para esquizofrenia, 15 $(12,1 \%)$ foram classificados como tendo psicose esquizofreniforme e $7(5,6 \%)$ como esquizoafetivos. Na avaliação global do ajustamento social, 57 sujeitos foram classificados como tendo ajustamento pobre ou ajustamento muito pobre, e os outros 57 sujeitos estavam igualmente distribuídos entre as classificações ajustamento excelente ou muito bom, bom ou razoável.

Dois anos depois, observou-se uma redução pela metade na proporção de sujeitos apresentando síndrome nuclear (delírios e alucinações auditivas), e quase $1 / 4$ dos pacientes não tinham nenhum sintoma psiquiátrico. Apenas a presença de um baixo nível educacional (menos de 4 anos de educação formal) e o diagnóstico de esquizofrenia segundo o DSM-III-R estiveram associados à presença de sintomas psicóticos na avaliação de seguimento. Um terço dos pacientes apresentava bom ajustamento social, outro terço tinha ajustamento intermediário e o último ajustamento pobre, resultado similar ao observado na avaliação de inclusão. As áreas de funcionamento social, em que os pacientes mostraram maiores proporções de melhora em relação à avaliação índice, foram retraimento social e cuidado pessoal. As características associadas a um maior risco de apresentar ajustamento social pobre, dois anos após a internação, foram maior tempo de duração da doença, ajustamento social pobre na avaliação inicial e um baixo nível educacional (menos de 4 anos de educação formal). Mais de $60 \%$ dos pacientes foram reinternados pelo menos uma vez durante o período de seguimento, e $42 \%$ apresentaram má adesão ao tratamento ambulatorial. A mortalidade foi 8 vezes maior que o esperado para a população dessa faixa etária, principalmente devido ao elevado número observado de suicídios.

O nível educacional esteve associado à presença de sintomas psicóticos e aos níveis de ajustamento social após 2 anos de seguimento. Com base nos achados do IPSS em Cali, Colômbia, ${ }^{5}$ sugere-se que nos países de Terceiro Mundo os pacientes com níveis educacionais mais altos poderiam ter um prognóstico pior que os menos escolarizados, devido às demandas e às expectativas sociais mais altas sobre os com maior escolaridade. No entanto, São Paulo é um grande centro urbano, onde as expectativas sociais e o estresse são provavelmente similares àqueles encontrados em grandes centros de países industrializados. Além disso, em países em desenvolvimento, o nível educacional pode representar uma medida mais apropriada de estrato social que a renda per capita. É possível também que, em São Paulo, não adquir o conhecimento educacional mínimo deixe o indivíduo com poucas habilidades para lidar com a complexidade de viver em um grande centro urbano industrializado.

Torna-se claro a partir do presente estudo que também no Brasil a maioria dos pacientes internados em hospitais psiquiátricos tem sintomas psicóticos e incapacidade social dois anos após a internação. Consequientemente, têm necessidades em termos de atenção à saúde e à seguridade social. Nos países industrializados, as pessoas que sofrem de esquizofrenia representam um custo muito alto para a sociedade, em termos diretos e indiretos. ${ }^{22}$ Elas precisam de acomodação, renda, cuidado da saúde, reabilitação e suporte social. Para melhorar sua qualidade, os serviços comunitários de atenção à saúde mental para pacientes psiquiátricos graves têm que ser abrangentes e integrados, facilmente acessíveis, orientados para os usuários e monitorados rotineiramente. ${ }^{23}$ Devem cobrir áreas como resposta à crise, cuidado continuado e a distância, e cuidado diário, de forma a garantir resposta adequada às demandas clínicas e de ajustamento social dos pacientes esquizofrênicos ao longo da evolução da doença. 


\section{Referências}

1. Biehl H, Maurer K, Schubart C, Krumm B, Jung E. Prediction of outcome and utilisation of medical services in a prospective study of first onset schizophrenics. Eur Arch Psychiatry Neurol Sci 1986;236:139-47.

2. Jablensky A, Sartorius N, Ernberg G, Anker M, Korten A, Cooper JE, et al. Schizophrenia: manifestations, incidence and course in different cultures. A World Health Organization ten-country study. Psychol Med 1992;Supl 20:1-97.

3. Scottish Schizophrenia Research Group. The scottish first episode schizophrenia study. VIII. Five-year follow-up: clinical and psychosocial findings. Br J Psychiatry 1992;161:496-500.

4. Shepherd M, Watt DC, Falloon I, Smeeton N. The natural history of schizophrenia: a five-year follow-up study of outcome and prediction in a representative sample of schizophrenics. Psychol Med 1989;Supl 15:1-46.

5. World Health Organization. Schizophrenia: an international followup study. Chichester: John Wiley \& Sons; 1979.

6. Harding CM, Brooks GW, Ashikaga T, Strauss JS, Breier A. The Vermont longitudinal study of persons with severe mental illness. Am J Psychiatry 1987;144:727-35.

7. Marneros A, Deister A, Rohde A. Psychopathological and social status of patients with affective, schizophrenic and schizo-affective disorders after long-term course. Acta Psychiatr Scand 1990;82:352-8.

8. Ogawa K, Miya M, Watarai A, Nakazawa M, Yuasa S, Utena H. A long-term follow-up study of schizophrenia in Japan: with special reference to the course of social adjustment. Br J Psychiatry 1987; 151:758-65.

9. Breier A, Schreiber JL, Dyer J, Pickar D. National Institute of Mental Health longitudinal study of chronic schizophrenia. Prognosis and predictors of outcome. Arch Gen Psychiatry 1991;48:239-46.

10.Carone BJ, Harrow M, Westermeyer JF. Posthospital course and outcome in schizophrenia. Arch Gen Psychiatry 1991;48:247-53.

11.De Jong A, Giel R, Slooff CJ, Wiersma D. Social disability and outcome in schizophrenics patients. Br J Psychiatry 1985;147:631-6.
12.Cooper JE, Bostock J. Relationship between schizophrenia, social disability, symptoms and diagnosis. In: Henderson, Burrows, editors. Handbook of Social Psychiatry. London: Elsevier Science Publishers; 1988. p. 317-30.

13.Geddes J, Mercer G, Frith CD, MacMillan F, Owens DGC, Johnstone EC. Prediction of outcome following a first episode of schizophrenia. Br J Psychiatry 1994;165:664-8.

14. Haro JM, Eaton WW, Bilker W, Mortensen PB. Predictability of rehospitalization for schizophrenia. Eur Arch Psychiatry Clin Neurosci 1994;244:241-6.

15. Mortensen PB, Eaton WW. Predictors for readmission risk in schizophrenia. Psychol Med 1994;24:223-32.

16.Black DW, Warrack G, Winokur G. Excess mortality among psychiatric patients. The Iowa Record-Linkage Study. JAMA 1985;253:58-61.

17.Mortensen PB, Juel K. Mortality and causes of death in first admitted schizophrenic patients. Br J Psychiatry 1993;163:183-9.

18. Newman SC, Bland RC. Mortality in a cohort of patients with schizophrenia: a record linkage study. Can J Psychiatry 1991;36:239-45.

19.Menezes PR. The outcome of schizophrenia and related psychoses after hospitalization: a prospective study in Sao Paulo, Brazil [dissertation]. London: London University; 1995.

20.Menezes PR, Mann AH. Mortality among patients with nonaffective functional psychoses in the city of São Paulo. Rev Saúde Pública 1996;30:304-9.

21. Menezes PR, Rodrigues LC, Mann AH. Predictors of clinical and social outcomes after hospitalization in schizophrenia. Eur Arch Psychiatry Clin Neurosci 1997;247:137-45.

22.Davies LM, Drummond MF. Economics and schizophrenia: the real cost. Br J Psychiatry 1994;165(Supl 25):18-21.

23. Strathdee G, Thornicroft G. The principles of setting up mental health services in the community. In: Bhugra D, Leff J, editors. Principles of Social Psychiatry. Oxford: Blackwell Scientific Publications; 1993. p.473-489.
Correspondência: Paulo Rossi Menezes

Departamento de Medicina Preventiva da FMUSP

Av. Dr. Arnaldo, 455

CEP 01246-903 São Paulo, SP - E-mail: pmenezes@usp.br 